EPJ Web of Conferences 71, 00013 (2014)

DOI: $10.1051 /$ epjconf / 20147100013

(C) Owned by the authors, published by EDP Sciences, 2014

\title{
Differential top-quark pair production measurements
}

\author{
Clare Bernard ${ }^{1, a}$ \\ ${ }^{1}$ Boston University
}

\begin{abstract}
The large number of top quarks produced at the CERN Large Hadron Collider makes it possible to measure the top-quark pair production cross-section differentially with respect to different observables. Differential measurements are important for precision tests and comparisons with Monte Carlo simulations and theoretical calculations. Top-quark pair production in particular is also an important process to study because it is a large background for many analyses. In this paper, several top-quark pair production differential measurements, performed with the ATLAS detector, are described including measurements of the kinematics of the top-quark pair system, of the jet multiplicity, and of the jet activity accompanying the top-quark pair system.
\end{abstract}

\section{Introduction}

The cross-section for top-quark pair production in $p p$ collisions at the LHC, $\sigma_{t \bar{t}}=177_{-11}^{+10} \mathrm{pb}$ at $\sqrt{s}=7 \mathrm{TeV}$ [1], is about a factor of 20 larger than it was at the Tevatron. This large increase in statistics means that more precise differential cross-section measurements are possible. These enable test of Monte Carlo simulations, theoretical predictions, and can provide data-driven constraints for various modeling systematic effects.

The ATLAS detector, described in detail elsewhere [2], is a multipurpose particle detector operating at the Large Hadron Collider. The ATLAS physics goals include making Standard Model measurements and searching for new particles or evidence of physics beyond the Standard Model.

This paper will review three ATLAS differential cross-section analyses using proton-proton collision data at a center-of-mass energy of $7 \mathrm{TeV}$. The first two analyses focus on the $t \bar{t}$ lepton+jets channel in which both top quarks decay to $W$ bosons and $b$ quarks and then one of the $W$ bosons decays to a lepton and a neutrino and the other decays to two quarks. The first measures the differential cross-section with respect to the invariant mass $\left(m_{t \bar{t}}\right)$, rapidity $\left(y_{t \bar{t}}\right)$, and transverse momentum $\left(p_{T}^{t \bar{t}}\right)$ of the $t \bar{t}$ system [3] while the second measures the jet multiplicity in $t \bar{t}$ events [4]. The third analysis focuses on the $t \bar{t}$ dilepton channel in which both $W$ bosons decay to leptons and measures jet activity accompanying the $t \bar{t}$ system [5].

\section{Differential cross-section measurements with respect to $t \bar{t}$ system kinematics}

The differential cross-sections for $t \bar{t}$ pair production with respect to $m_{t \bar{t}}, y_{t \bar{t}}$, and $p_{T}^{t \bar{t}}$ were measured by ATLAS using data collected at $7 \mathrm{TeV}$ in 2011 with an integrated luminosity of $2.0 \mathrm{fb}^{-1}$. The observed

\footnotetext{
a e-mail: clare.bernard@ cern.ch
} 
Figure 1. The $-\ln$ (likelihood) in the electron channel. Data points are compared to the prediction obtained from a combination of Monte Carlo simulation and data-driven techniques. The hashed black $\triangle$ lines denote the combined statistical and systematic uncertainty [3].

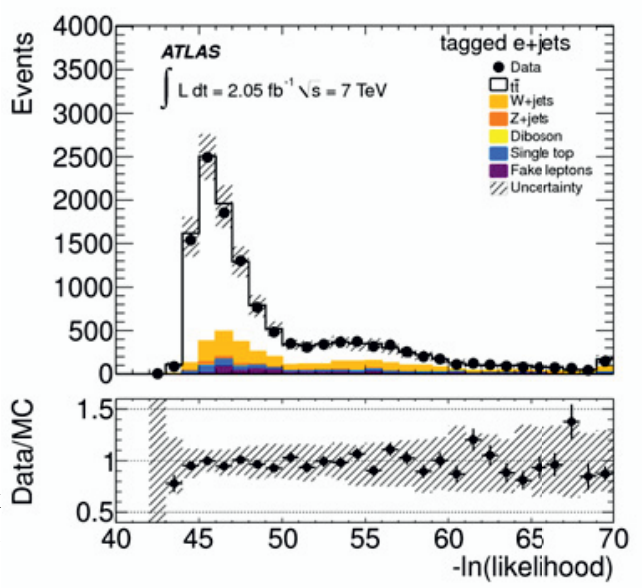

spectra were compared to the predictions from different Monte Carlo generators [3] and $m_{t \bar{t}}$ and $y_{t \bar{t}}$ were compared to predictions determined using different parton distribution function (PDF) sets at NLO [6].

\subsection{Event Selection and Reconstruction of the $t \bar{t}$ System}

In order to select events in the lepton+jets channel, events are required to have exactly one lepton (electron or muon), at least four jets, of which at least one is identified as originating from a $b$ quark (tagged). Electrons are required to have $E_{T}>25 \mathrm{GeV}$, muons are required to have $p_{T}>20 \mathrm{GeV}$, and jets are required to have $p_{T}>25 \mathrm{GeV}$. In addition, requirements are placed on the missing transverse momentum $\left(E_{T}^{m i s s}\right)$ and the transverse $W$ boson mass $\left(m_{T}^{W}\right)$ which is defined as

$$
m_{T}^{W}=\sqrt{2 p_{T}^{\ell} p_{T}^{v}\left(1-\cos \left(\phi^{\ell}-\phi^{v}\right)\right)}
$$

where $p_{T}^{v}$ and $\phi^{v}$ are calculated from the direction and magnitude of $E_{T}^{\text {miss }}$. The requirements placed on the electron channel are stronger due to the need to reduce the larger fake-lepton background.

The $t \bar{t}$ system is reconstructed using a kinematic likelihood fitter which uses as input the five jets with the highest $p_{T}$ as well as the energy and direction of the lepton, the $E_{T}^{\text {miss }}$, and the $b$-tagging information. Out of the possible jet permutations, the one that best suits the $t \bar{t}$ decay topology is retained. The likelihood function includes Breit-Wigner terms which constrain the reconstructed $W$ boson and top quark to be near their known masses, and transfer functions derived from Monte Carlo which related the measured energies of the jets to the decay products of the $t \bar{t}$ system. The observed and predicted likelihood distribution is shown for the $e+$ jets channel in Fig. 1. A requirement is made that the variable plotted, $-\ln ($ likelihood), be less than 52 in order to promote well-reconstructed events.

To perform differential measurements as a function of a given kinematic quantity, the background contributions must be subtracted. The largest background to this measurement originates from $W$ boson production. This is referred to as $W+$ jets in Fig. 1. Other significant backgrounds originate from $Z$ bosons, dibosons, single top quark, and the multijet background (referred to as fake leptons). The $W+$ jets and multijet background are determined using data-driven techniques while the other backgrounds are all estimated from Monte Carlo simulations. 


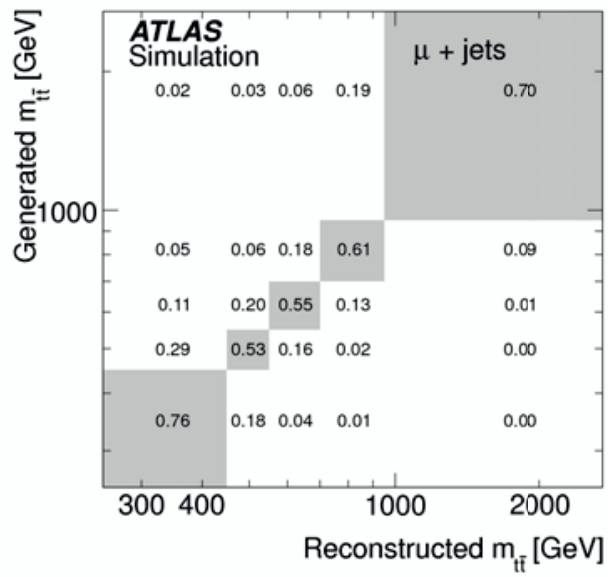

Figure 2. The migration matrix in the muon channel relating the reconstructed $m_{t \bar{t}}$ to the truth level $m_{t \bar{t}}$. The numbers in the diagonal bins show the fraction of events that stay in the same bin and the off diagonal bins show the migrations to other bins [3].

\subsection{Unfolding}

To compare to theoretical calculations, detector acceptance and resolution effects have to be corrected using an unfolding procedure. This can be done by constructing a migration matrix which relates the reconstructed level information to the truth level information using Monte Carlo simulation. The migration matrix in the $\mu+$ jets channel is shown in Fig 2.

This analysis uses an unregularized unfolding method, inverting the migration matrix, and applying it to the measured spectra [7]. The measured spectra are also corrected to account for the branching ratio from $t \bar{t}$ to decay in the lepton+jets channel, and the efficiency for events to pass all requirements mentioned in Section 2.

After unfolding, the electron and muon channels are combined using the Best Linear Unbiased Estimator method. The final results are normalized to the integrated cross-section to reduce the total uncertainty.

\subsection{Results}

The final unfolded results are shown for the $m_{t \bar{t}}$ and $p_{T}^{t \bar{t}}$ spectra in Fig. 3. The measured spectra are compared with predictions from the ALPGEN [8] and MC@NLO [9] generators and from NLO QCD predictions and all spectra agree within uncertainties. These generators are interfaced with HERWIG [10] and JIMMY [11] for parton showering.

The $m_{t \bar{t}}$ and $y_{t \bar{t}}$ spectra are also compared with predictions at NLO using different PDF sets interfaced with MCFM [12]. The PDF sets vary for a variety of reasons: the value of the strong coupling constant, the fitting procedure, and the input data used. For example, CT10 [13], NNPDF2.3 [14], and HERA PDF 1.5 [15] use $\alpha_{S}=0.118,0.118$, and 0.1176 respectively while MSTW 2008 [16] and ABM 11 [17] determine $\alpha_{S}$ simultaneously as part of the fit and get values of 0.1202 and 0.1134 . Comparisons to these different PDF sets are shown in Fig. 4. Some differences between different PDF sets are observed, but the uncertainties on the measured spectra presented in this paper are too large to discriminate. The $m_{t \bar{t}}$ and $y_{t \bar{t}}$ variables were chosen for this comparison because they were observed to have the greatest sensitivity to the effects of different PDF sets. 

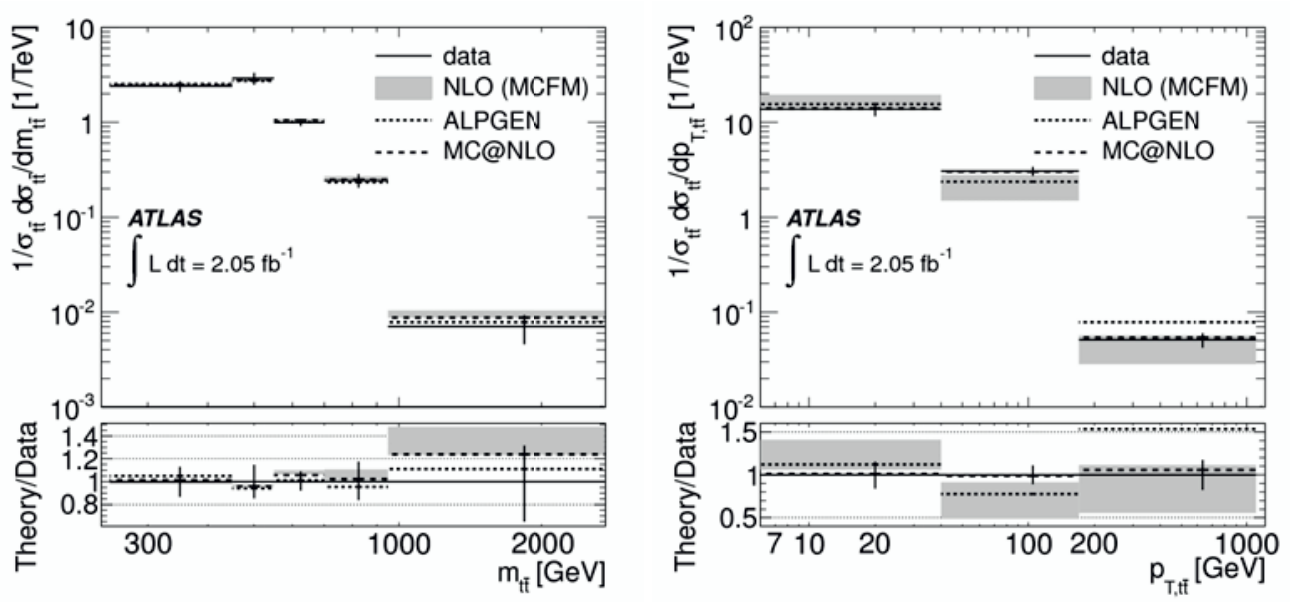

Figure 3. The unfolded $m_{t \bar{t}}$ (left) and $p_{T}^{t \bar{t}}$ (right) spectra. The grey bands denote the uncertainty on the NLO prediction, while the error bars represent the combined statistical and systematic uncertainty on the measured spectra [3].
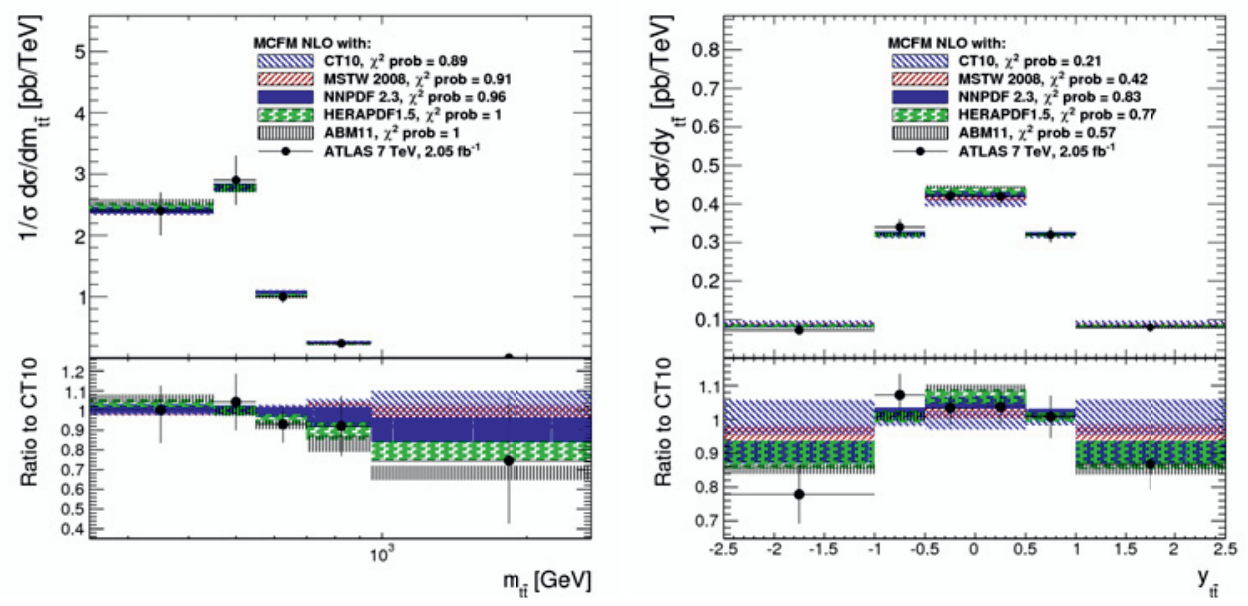

Figure 4. The unfolded $m_{t \bar{t}}$ (left) and $y_{t \bar{t}}$ (right) spectra compared with NLO QCD predictions using different PDF sets. The colored bands represent the PDF uncertainties while the error bars on the data points represent the combined statistical and systematic uncertainties on the measured spectra [6].

\section{Jet multiplicity measurement}

The jet multiplicity spectra in $t \bar{t}$ events were measured differentially with the ATLAS detector using the full $7 \mathrm{TeV} 2011$ dataset corresponding to an integrated luminosity of $4.7 \mathrm{fb}^{-1}$ [4]. The jet multiplicity is measured for jets over four different $p_{T}$ thresholds: $25,40,60$ and $80 \mathrm{GeV}$, using a kinematic selection very close to that described in Sec. 2.1. The measured jet multiplicity is shown in the $e+$ jets channel for jets above $40 \mathrm{GeV}$ in Fig. 5. The largest systematic uncertainties come from the jet 


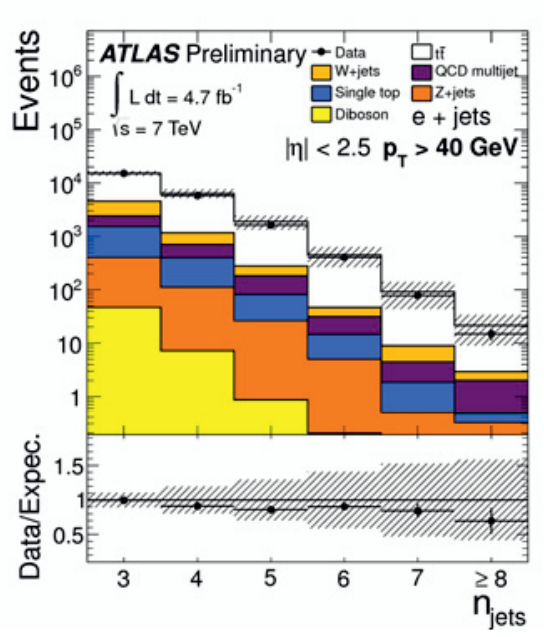

Figure 5. The measured jet multiplicity distribution in the electron channel for jets with $p_{T}>40 \mathrm{GeV}$. ALPGEN+HERWIG is used for the signal generator and the hashed black lines denote the combined statistical and systematic uncertainty [4].

energy scale at high jet multiplicity, and from the uncertainty on the background predictions at low jet multiplicity.

Unlike the previous measurement which was corrected to obtain a full phase-space result, the jet multiplicity spectrum was corrected to particle-level, obtaining a fiducial cross-section measurement. A migration matrix was constructed, and the observed spectra were unfolded iteratively using the Bayesian method [18].

\subsection{Results}

Final distributions are shown in the muon channel after background subtraction and unfolding for jets above $p_{T}$ thresholds of 25 and $80 \mathrm{GeV}$ in Fig. 6. Good agreement is observed with respect to the predictions from several generators (ALPGEN+HERWIG, ALPGEN+PYTHIA [19], and POWHEG [20]+PYTHIA) while MC@NLO+HERWIG predicts too few jets in the high multiplicity bins.

\section{Measurement of $t \bar{t}$ production with a veto on additional central jet activity}

The jet activity in $t \bar{t}$ events was measured differentially in the dilepton channel with the ATLAS detector using a data collected at a center-of-mass energy of $7 \mathrm{TeV}$ in 2011 with an integrated luminosity of $2.05 \mathrm{fb}^{-1}$. This measurement as well as the previous measurement of jet multiplicity can be used to constrain systematic uncertainties due to the theoretical description of quark and gluon radiation accompanying the $t \bar{t}$ system. The fraction of events which do not contain an extra jet above a $p_{T}$ threshold $Q_{0}$ or that do not contain extra jets whose $p_{T}$ scalar sum exceeds $Q_{\text {sum }}$ can be defined as:

$$
f\left(Q_{0}\right)=\frac{n\left(Q_{0}\right)}{N}=\frac{\sigma\left(Q_{0}\right)}{\sigma}, \quad f\left(Q_{\text {sum }}\right)=\frac{n\left(Q_{\text {sum }}\right)}{N}=\frac{\sigma\left(Q_{\text {sum }}\right)}{\sigma}
$$

where $\mathrm{N}$ is the total number of events. In this context, $f\left(Q_{0}\right)$ is sensitive to the leading jet momentum while $f\left(Q_{\text {sum }}\right)$ is sensitive to all jet activity. Both are measured as fiducial cross-sections. 

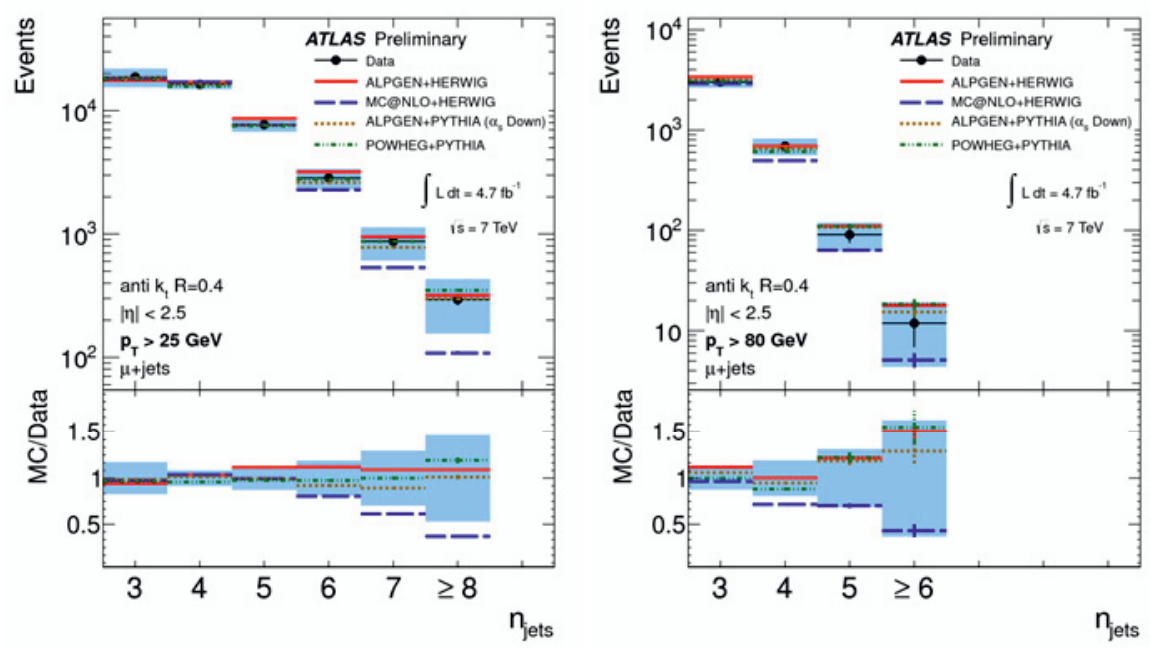

Figure 6. Unfolded jet multiplicity distributions in the muon channel for jets with $p_{T}$ above $25 \mathrm{GeV}$ (left) and $80 \mathrm{GeV}$ (right). The measured distributions are compared with the predictions from several generators: ALPGEN+HERWIG, MC@NLO+HERWIG, ALPGEN+PYTHIA, and POWHEG+PYTHIA [4].

\subsection{Event Selection}

The analysis is based on the dilepton $t \bar{t}$ channel: two oppositely charged high $p_{T}$ leptons are required as well as at least two b-tagged jets. Additional requirements are made depending on the flavors of the leptons. For events with two electrons or two muons significant $E_{T}^{m i s s}$ is required and the invariant mass of the lepton pair must be greater than $15 \mathrm{GeV}$ and must not be near the $Z$ boson mass. For events with one electron and one muon the $H_{T}$ for the event, defined as the scalar sum of the transverse momenta of all jets and leptons, is required to be greater than $130 \mathrm{GeV}$.

The measured jet veto distribution is unfolded by applying a bin-by-bin correction factor $\mathrm{C}(\mathrm{x})$ where x is either $Q_{0}$ or $Q_{\text {sum }}$

$$
C(x)=\frac{f^{\text {truth }}(x)}{f^{\text {reco }}(x)}
$$

These fractions are determined from Monte Carlo simulations. The dominant sources of systematic uncertainties for this measurement come from jet energy scale, jet energy resolution and the uncertainty associated with these correction factors.

\subsection{Results}

The final results for $f\left(Q_{0}\right)$ in the rapidity intervals $1.5 \leq|y|<2.1$ and $|y|<2.1$ are shown in Fig. 7 and the final result for $f\left(Q_{\text {sum }}\right)$ in the interval $|y|<0.8$ is shown in Fig. 8. For both $f\left(Q_{0}\right)$ and $f\left(Q_{\text {sum }}\right)$ good agreement is found between the measured spectra and predictions over the full rapidity interval. However, all models are found to overestimate the amount of extra radiation in the forward detector region and MC@NLO+HERWIG in particular, produces too little activity in the central region. Comparisons with the AcerMC generator [21] such as in Fig. 8 were able to greatly reduce the systematic uncertainties associated with initial and final state radiation in $t \bar{t}$ analyses. 

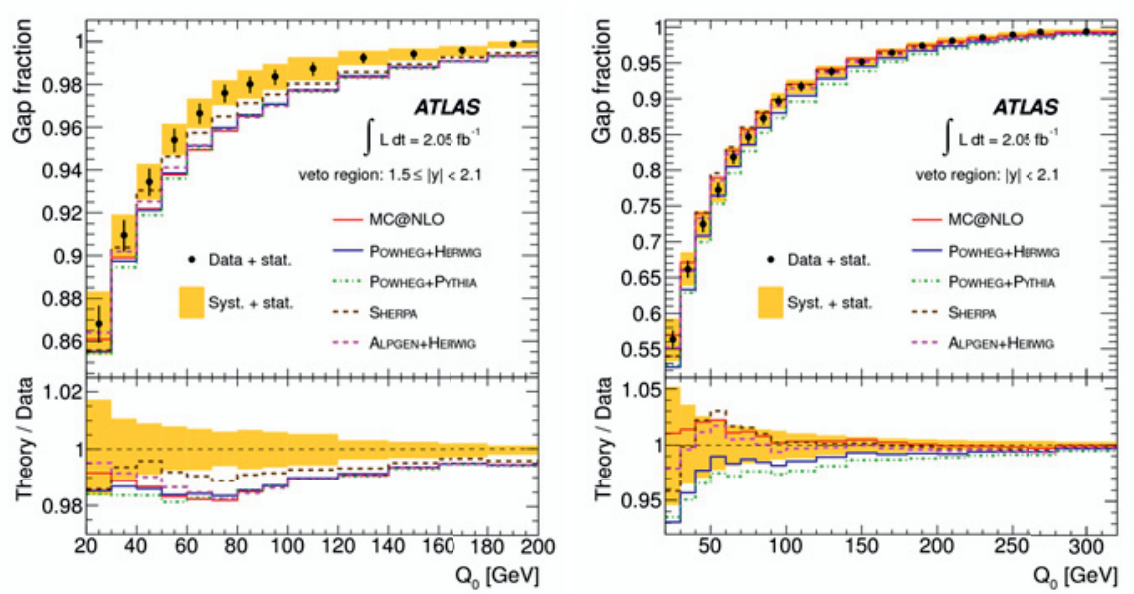

Figure 7. The unfolded jet veto measurement for $f\left(Q_{0}\right)$ in the rapidity intervals $1.5 \leq|y|<2.1$ (left) and $|y|<2.1$ (right). The yellow band represents the combined statistical and systematic uncertainties on the measurement and comparisons are shown to several different Monte Carlo generators [5].

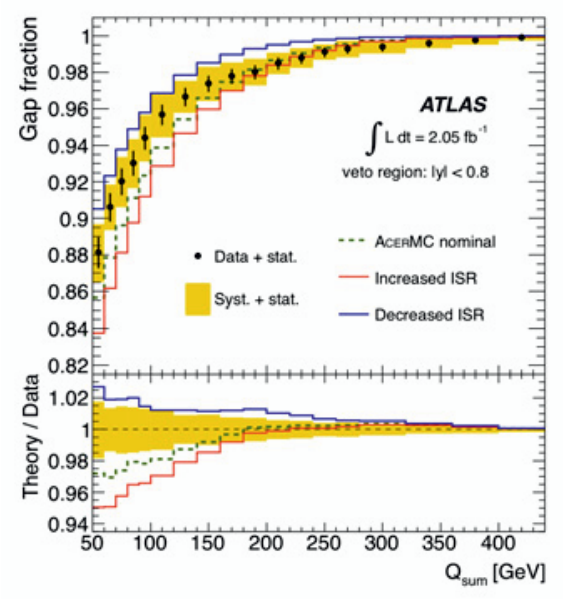

Figure 8. The unfolded jet gap fraction measurement for $f\left(Q_{\text {sum }}\right)$ in the central rapidity interval $|y|<0.8$. The yellow band represents the combined statistical and systematic uncertainties on the measurement and comparisons are shown with the AcerMC generator using different settings for the PYTHIA parton shower parameters [5].

\section{Conclusion}

Several $t \bar{t}$ differential cross-section measurements have been made using proton-proton collision data at a center-of-mass energy of $7 \mathrm{TeV}$ collected by the ATLAS detector. These are key results to test theoretical predictions and improve the modeling and related uncertainty of top-quark pair signal events in Monte Carlo simulation. In addition these measurements constitute a promising window for searching for beyond the Standard Model physics effects.

\section{References}

[1] M. Cacciari, M. Czakon, M. Mangano, A. Mitov, P. Nason, Phys. Lett. B710, 612 (2012)

[2] ATLAS Collaboration, JINST 3, S08003 (2008) 
[3] ATLAS Collaboration, Eur. Phys. J. C73, 2261 (2013)

[4] ATLAS Collaboration (2012), ATLAS-CONF-2012-155

[5] ATLAS Collaboration, Eur. Phys. J. C72, 2043 (2012)

[6] ATLAS Collaboration (2013), ATL-PHYS-PUB-2013-008

[7] V. Blobel, p. 40 p (1984), DESY-84-118

[8] M.L. Mangano, M. Moretti, F. Piccinini, R. Pittau, A.D. Polosa, JHEP 0307, 001 (2003)

[9] S. Frixione, P. Nason, B.R. Webber, JHEP 0308, 007 (2003)

[10] G. Corcella, I. Knowles, G. Marchesini, S. Moretti, K. Odagiri et al., JHEP 0101, 010 (2001)

[11] J. Butterworth, J.R. Forshaw, M. Seymour, Z. Phys. C72, 637 (1996)

[12] J. Campbell, K. Ellis, C. Williams, http://mcfm.fnal.gov/

[13] J. Gao, M. Guzzi, J. Huston, H.L. Lai, Z. Li et al. (2013), SMU-HEP-12-23

[14] R.D. Ball, V. Bertone, S. Carrazza, C.S. Deans, L. Del Debbio et al., Nucl. Phys. B867, 244 (2013)

[15] A. Cooper-Sarkar (ZEUS Collaboration, H1 Collaboration), PoS EPS-HEP2011, 320 (2011)

[16] A. Martin, W. Stirling, R. Thorne, G. Watt, Eur. Phys. J. C63, 189 (2009)

[17] S. Alekhin, J. Bluemlein, S.O. Moch, PoS LL2012, 016 (2012)

[18] G. D’Agostini, Nucl. Instrum. Meth. A362, 487 (1995), DESY-94-099

[19] T. Sjostrand, S. Mrenna, P.Z. Skands, JHEP 0605, 026 (2006)

[20] S. Alioli, P. Nason, C. Oleari, E. Re, JHEP 1006, 043 (2010)

[21] B.P. Kersevan, E. Richter-Was (2004), TPJU-6-2004 